Abstract THU0346 -Table 1.

\begin{tabular}{|l|l|l|l|l|}
\hline Analyte & IIM Subtype & Sensitivity $(95 \% \mathrm{Cl})$ & Specificity $(95 \% \mathrm{Cl})$ & Odds Ratio $(95 \% \mathrm{Cl})$ \\
\hline Jo-1 & ASS $(\mathrm{n}=67)$ & $71.6 \%(59.9-81.0 \%)$ & $98.0 \%(94.9-99.2 \%)$ & $121.9(40.8-360.0)$ \\
\hline PL-7 & ASS & $10.4 \%(5.2-20.0 \%)$ & $100.0 \%(98.1-100.0 \%)$ & $+\infty(5.8-+\infty)$ \\
\hline PL-12 & ASS & $7.5 \%(3.2-16.3 \%)$ & $100.0 \%(98.1-100.0 \%)$ & $+\infty(4.0-+\infty)$ \\
\hline EJ & ASS & $3.0 \%(0.8-10.2 \%)$ & $99.5 \%(97.2-99.9 \%)$ & $6.0(0.7-46.8)$ \\
\hline Mi-2 & DM $(n=70)$ & $21.4 \%(13.4-32.4 \%)$ & $97.9 \%(94.8-99.2 \%)$ & $13.0(4.3-38.7)$ \\
\hline NXP2 & DM & $7.1 \%(3.1-15.7 \%)$ & $98.5 \%(95.6-99.5 \%)$ & $4.9(1.3-19.1)$ \\
\hline SAE & DM & $11.4 \%(5.9-21.0 \%)$ & $99.0 \%(96.3-99.7 \%)$ & $12.4(2.9-52.9)$ \\
\hline TIF1y & DM & $15.7 \%(9.0-26.0 \%)$ & $96.2 \%(92.0-98.3 \%)$ & $4.8(1.7-13.0)$ \\
\hline TIF1y & CDM $(n=35)$ & $62.9 \%(46.3-76.8 \%)$ & $96.2 \%(92.0-98.3 \%)$ & $43.2(15.1-122.8)$ \\
\hline MDA5 & CADM $(\mathrm{n}=21)$ & $71.4 \%(50.0-86.2 \%)$ & $97.1 \%(94.2-98.6 \%)$ & $84.3(25.7-277.5)$ \\
\hline HMGCR & IMNM $(\mathrm{n}=16)$ & $50.0 \%(28.0-72.0 \%)$ & $96.8 \%(93.8-98.4 \%)$ & $30.0(9.2-98.2)$ \\
\hline
\end{tabular}

ASS = Anti-Synthetase Syndrome, DM = Dermatomyositis, $C D M=$ Cancer Associated Dermatomyositis, CADM = Amyopathic Dermatomyositis

Results: The sensitivity/specificity of the individual MSA were: $19.7 \% /$ 100\% (Jo-1), $\quad 7.2 \% / 100.0 \% \quad$ (Mi-2), $3.0 \% / 99.0 \% \quad$ (NXP2), $3.8 \% / 100.0 \%$ (SAE), 2.7\%/100.0\% (PL-7), 1.9\%/99.5 (PL-12), 1.1\%/100.0\% (EJ), 15.5\%/ $99.5 \%$ (TIF1y), 8.3\%/98.5\% (MDA5), 6.1\%/99.0\% (HMGCR) and $1.9 \% /$ $98.5 \%$ (SRP). The overall clinical performance was: sensitivity $68.2 \%$ (95\% confidence interval $62.3-73.5 \%)$, specificity $94.0 \% \quad(95 \% \mathrm{Cl} \quad 89.8-$ $96.5 \%$ ) and odds ratio 33.8 . In the table below, the sensitivity and specificity of each analyte for IIM subtypes was calculated along with odds ratio.

Conclusion: The novel PMAT used to detect a spectrum of MSA in IIM on a fully automated system showed good sensitivity and specificity in line with the known associations of MSA. Sensitivities and specificities of the individual MSA are within expected ranges. Lastly, the individual markers help to stratify patients into IIM subtype which is important for management of the patients.

Disclosure of Interests: Michael Mahler Employee of: Inova Diagnostics (Not pharmaceutical, diagnostics company), Kishore Malyavantham Employee of: Inova Diagnostics, Michaelin Richards Employee of: Inova Diagnostics, Chelsea Bentow Employee of: INOVA Diagnostics, Silvia Casas Employee of: Inova Diagnostics, Eva Balada: None declared, Maite Sanz: None declared, M. Angeles Martinez: None declared, Albert SelvaO'Callaghan: None declared

DOI: 10.1136/annrheumdis-2019-eular.8101

\section{THU0347 TESTING THE IN VITRO EFFECTS OF NINTEDANIB ON CIRCULATING FIBROCYTES AND RESIDENT SKIN FIBROBLASTS FROM THE SAME SYSTEMIC SCLEROSIS PATIENTS: PRELIMINARY RESULTS}

Maurizio Cutolo ${ }^{1}$, Paola Contini ${ }^{2}$, Giulia Martinelli ${ }^{1}$, Paola Montagna ${ }^{1}$, Samuele Tardito ${ }^{1}$, Sabrina Paolino ${ }^{1}$, Massimo Patanè ${ }^{1}$, Carmen Pizzorni ${ }^{1}$, Elisa Alessandri ${ }^{1}$, Veronica Tomatis ${ }^{1}$, Vanessa Smith ${ }^{3,4,5}$, Stefano Soldano ${ }^{1}$ ${ }^{1}$ University of Genova, IRCCS Polyclinic San Martino Hospital, Research Laboratory and Academic Division of Clinical Rheumatology, Dept. of Internal Medicine, Genoa, Italy, ${ }^{2}$ University of Genova, IRCCS Polyclinic San Martino Hospital, 2 Clinical Immunology, Dept. of Internal Medicine, Genoa, Italy; ${ }^{3}$ Ghent University Hospital, Dept. of Rheumatology, Ghent University Hospital, Ghent, Belgium; Dept. of Internal Medicine, Ghent, Belgium; ${ }^{4}$ Ghent University, Dept. of Internal Medicine, Ghent, Belgium; ${ }^{5}$ VIB Inflammation Research Center (IRC), Unit for Molecular Immunology and Inflammation, Ghent, Belgium

Background: The fibrosis in systemic sclerosis (SSc) progresses from microvascular alterations, immune system activation, and increased extracellular matrix protein synthesis into the skin and internal organs, primarily mediated by myofibroblasts. ${ }^{1}$ Myofibroblasts are characterized by a higher expression of $\alpha$-smooth muscle actin $(\alpha S M A)$ and by the overproduction of type I collagen (COL1) and fibronectin (FN). ${ }^{2}$ Although myofibroblasts primarily derive from fibroblasts differentiation and transition, circulating fibrocytes represent a further important source. ${ }^{3}$ Nintedanib is a tyrosine kinase inhibitor that interacts with several inflammatory and profibrotic pathways implicated in the pathogenesis of fibrosis, including those of platelet derived growth factor receptors, vascular endothelial growth factor receptors and fibroblast growth factor receptors. ${ }^{4}$

Objectives: To investigate, in primary cultures, the effects of nintedanib on the differentiation of circulating fibrocytes and on the profibrotic activity of skin fibroblasts isolated from the same SSc patients.

Methods: Circulating fibrocytes and fibroblasts were obtained from peripheral blood and skin biopsies, respectively, from three untreated SSc patients with diffuse skin involvement (mean age $55 \pm 6 \mathrm{yrs}$ ). To investigate the complete differentiation, fibrocytes were maintained in DMEM at $20 \%$ of foetal bovine serum, either with or without treatment with nintedanib at the concentrations of $0.1 \mu \mathrm{M}$ and $1 \mu \mathrm{M}$ for 8 days. ${ }^{5}$ Fibrocytes were characterized as $\mathrm{CD} 45^{+} \mathrm{CXCR} 4^{+} \mathrm{COL} 1^{+}$cells, and their percentage was detected by Flow Cytometry analysis. ${ }^{6}$ Fibroblasts were grown until the $3^{\text {dr }}$ culture passage and then maintained in normal growth medium with or without nintedanib treatment for 4,24 and 48 hours. The gene expressions of $\alpha$ SMA, COL1 and FN were evaluated by qRT-PCR.

Results: Nintedanib reduced the percentage of differentiated SSc fibrocytes $\left(\mathrm{CD} 45^{+} \mathrm{CXCR} 4^{+} \mathrm{COL} 1^{+}\right.$cells), already at the concentration of $0.1 \mu \mathrm{M}$ compared with untreated fibrocytes. In cultured SSc skin fibroblasts, nintedanib $1 \mu \mathrm{M}$ downregulated $\alpha \mathrm{SMA}, \mathrm{COL} 1$ and $\mathrm{FN}$ expressions already after 4 hours of treatment, and this effect was also maintained after 24- and 48-hours' treatment. Nintedanib $0.1 \mu \mathrm{M}$ downregulated the $\alpha \mathrm{SMA}$ expression at all timepoints, whereas the downregulation of COL1 and FN expressions was observed at 4 and 24 hours, with no more effect at 48 hours of treatment compared with untreated cells.

Conclusion: Initial experiments seem to indicate an evident in vitro concentration-dependent antifibrotic activity of nintedanib on SSc fibrocytes and skin fibroblasts from the same SSc patients; in particular by reducing the fibrocyte differentiation as potential source of myofibroblasts and con trasting the profibrotic activity of already activated fibroblasts/myofibroblasts. These results have translational implications in clinical trials using nintedanib in SSc patients.

\section{REFERENCES}

[1] Furue M, et al. Immunol Res 2017;65:790-7.

[2] Kendall RT, et al. Front Pharmacol 2014;5:123

[3] Strieter RB, et al. J Leukocyte Biol 2009;88:111-8.

[4] Huang J, et al. Ann Rheum Dis 2017;76:1941-8.

[5] Cutolo M, et al. Arthritis Res Ther 2018;20:157.

Disclosure of Interests: None declared

DOI: 10.1136/annrheumdis-2019-eular.6909

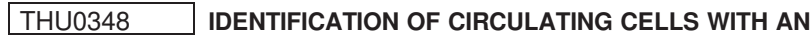 HYBRID M1/M2 MACROPHAGE PHENOTYPE IN SYSTEMIC SCLEROSIS PATIENTS AND CORRELATIONS WITH SELECTED CLINICAL ASPECTS}

Stefano Soldano ${ }^{1}$, Amelia Chiara Trombetta ${ }^{1}$, Paola Contini $^{2}$, Greta Pacini ${ }^{1}$, Veronica Tomatis ${ }^{1}$, Paola Montagna ${ }^{1}$, Massimo Patanè ${ }^{1}$, Elisa Alessandri ${ }^{1}$, Federica Goegan 1 , Vanessa Smith ${ }^{3,4}$, Maurizio Cutolo'. ' ${ }^{1}$ University of Genova, IRCCS Polyclinic San Martino Hospital, Research Laboratory and Academic Division of Clinical Rheumatology, Dept. of Internal Medicine, Genoa, Italy, ${ }^{2}$ University of Genova, IRCCS Polyclinic San Martino Hospital, Clinical Immunology, Dept. of Internal Medicine, Genoa, Italy; ${ }^{3}$ Ghent University, Ghent, Dept. of Rheumatology, Ghent University Hospital, Ghent, Belgium; Dept. of Internal Medicine, Ghent, Belgium; ${ }^{4}$ VIB Inflammation Research Center (IRC), Unit for Molecular Immunology and Inflammation, Ghent, Belgium

Background: Systemic sclerosis (SSc) is characterized by immune system alterations, vascular damage and fibrosis (1). Macrophages seem to have an emerging role in SSc, and the characterization of their polarized phenotype, starting from the dichotomic definition of classically activated (M1) or alternatively activated (M2) macrophages, is a recent research topic of interest (2)

Objectives: The study investigated a possible imbalance in the distribution of circulating cells expressing $\mathrm{M} 1$ and $\mathrm{M} 2$ markers in SSc patients (pts) compared to healthy subjects (HSs), and the presence of circulating cells co-expressing M1 and M2 surface markers. Possible correlations between their percentage and selected SSc clinical aspects were investigated.

Methods: In the study 55 SSc pts (50 females/5 males, mean age 64 $\pm 13 \mathrm{yrs}$ ), fulfilling the new EULAR/ACR criteria for SSc diagnosis, and 27 age-matched HSs (25 females/2 males, mean age $57 \pm 7$ yrs) were enrolled after written informed consent. Nailfold videocapillaroscopy (NVC), evaluation of SSc-related antibodies and pulmonary functional tests were performed. In particular, circulating cells belonging to the leukocyte and monocyte populations (CD $45^{+}$and $\mathrm{CD} 14^{+}$cells) were investigated by flow cytometry (FC) using the surface markers characterizing M1 (CD80 CD86, TLR2, TLR4) and M2 phenotypes (CD204, CD206, CD163). Statistical analysis was performed using Mann-Whitney and Kruskal-Wallis tests, and correlations were explored by bivariate Pearson's analysis.

Results: Increased circulating cells showing an M2 phenotype and characterized as $\mathrm{CD}_{20} 4^{+} \mathrm{CD} 206^{+} \mathrm{CD} 163^{+}$cells was observed in SSc pts compared to HSs $(p<0.0001)$, whereas no difference in CD80 ${ }^{+}$CD86 ${ }^{+} \mathrm{TLR}_{2}{ }^{+} \mathrm{TLR}^{+}{ }^{+}(\mathrm{M} 1)$ cell percentage was observed. A significant higher percentage of circulating cells showing a hybrid M1/M2 phenotype (CD204 ${ }^{+} \mathrm{CD} 206^{+} \mathrm{CD}_{163}{ }^{+} \mathrm{TLR}^{+} \mathrm{CD} 80^{+} \mathrm{CD} 86^{+}$cells) was observed in SSc pts compared to HSs. These hybrid M1/M2 cells were significantly increased in SSc pts either treated with steroids or under no immunosuppressive treatment $(p<0.01 ; p<0.05)$, as well as in Scl70 ${ }^{+} p t s \quad(p<0.05$ vs. Scl70 $p t s)$ 
and SSc pts with interstitial lung disease (ILD) and high systolic pulmonary artery pressure (PAP). A linear negative correlation between the high hybrid M1/M2 cell percentage and diffusing capacity of the lungs for carbon monoxide (DLCO)\% and the forced vital capacity (FVC)/DLCO ratio higher than 1.5 was observed. No significant correlations were reported with SSc duration, other treatments, NVC patterns, renal artery resistive index, heart and kidney involvements, digital ulcers, telangiectasias, calcinosis

Conclusion: The study identified a circulating cell population expressing both M1 and M2 surface markers, which is increased together with circulating $\mathrm{M} 2$ cells in SSc pts, in particular affected by ILD and high PAP, suggesting their possible involvement in the pathogenesis of those disease complications. Further evaluations are in progress.

\section{REFERENCES:}

[1] Cutolo M, et al. Nat Rev Rheumatol. 2015;11:569-71. 2. Stifano G, et al. Curr Rheumatol Rep. 2016;18:2.

Disclosure of Interests: None declared DOI: 10.1136/annrheumdis-2019-eular.6748

\section{THU0349 AUTOLOGOUS FAT GRAFTING IN THE TREATMENT OF PATIENTS WITH SYSTEMIC SCLEROSIS: CURRENT EXPERIENCE AND FUTURE PROSPECTS}

Amelia Spinella ${ }^{1}$, Marco Pignatti ${ }^{2}$, Giorgia Citriniti ${ }^{1}$, Federica Lumetti ${ }^{1}$, Emanuele Cocchiara ${ }^{1}$, Adalgisa Palermo ${ }^{1}$, Gianluca Sighinolfi ${ }^{1}$,

Lucrezia Pacchioni ${ }^{3}$, Giovanna Zaccaria ${ }^{3}$, Irene Laura Lusetti ${ }^{3}$, Giorgio De Santis ${ }^{3}$ Carlo Salvarani ${ }^{1}$, Dilia Giuggioli'. . 'Scleroderma Unit, Chair of Rheumatology, University of Modena and Reggio Emilia, Modena, Italy; ${ }^{2}$ Department of Experimental Diagnostic and Specialty Medicine, S. Orsola-Malpighi University Hospital, Modena, Italy; ${ }^{3}$ Department of Medical and Surgical Sciences, University of Modena and Reggio Emilia, Modena, Italy

Background: Systemic Sclerosis (SSc) is a connective tissue disease, characterized by endothelial dysfunction and fibrosis, potentially affecting internal organs and reducing life expectancy. Digital ulcers (DUs), as well as hand and face skin thickening, are the hallmarks of the disease. These alterations lead to pain, functional impairment, aesthetic damages, and psychological distress. Autologous fat grafting (AFG) is a surgical technique used also to promote tissue regeneration. In the last decade, AFG has been successfully developed to treat clinical conditions characterized by skin atrophy or fibrosis. AFG composition of multipotent cells, carrying angiogenic, and immunogenic properties, may be able to restore the damaged tissues.

Objectives: Evaluate our experience with AFG to treat and prevent damage and disability due to DUs and SSc skin complications.

Methods: We analyzed 25 SSc patients, extrapolated from a larger series of 45 subjects, complaining about mouth and/or hand impairment, due to skin involvement, and, in some cases, long-lasting DUs (M/F 6/19, mean age 55.69 \pm 9.25 -SD-years, mean disease duration 184.68 $\pm 121.09-S D$ months, L/D cutaneous subsets 21/4). Surgical procedures consisted in the injection of centrifuged and purified autologous fat, harvested from hips or abdomen. $2 \mathrm{ml}$ of fat were grafted in each of the 8 sites around the mouth, while 0.5 or $1 \mathrm{ml}$ around the neurovascular bundle at the base of each finger. The study included: preoperative data collection; 2 or 3 surgical sessions at a distance of 6 months one from the other; data collection at 3 months after each surgical session; data collection at 3 and 6 months of follow up-FU after the last surgical procedure. Data collection consisted of clinic-serological SSc features and clinimetric measures about hand and mouth, if present DUs were assessed as well. Furthermore, patients were asked to fill in questionnaires in order to express their level of satisfaction with hand and mouth functionality.

Results: Overall 63 surgical procedures were performed. After 1 to 3 procedures, patients reported an improvement of perioral skin tension $(p=0.0238)$ and a reduced dry mouth feeling $(p=0.0269)$. Similarly, patients stated an improvement of hands tension $(\mathrm{p}=0.0037)$. Furthermore, we observed a complete healing of DUs in $8 / 9$ patients $(p=0.0297)$. These positive clinical changes were mirrored by a subjective amelioration. In particular, pain decreased, evaluated by short-form McGill Pain Questionnaire (SF-MPQ), scrutinizing sensory $(\mathrm{p}=0.3340)$ and affective $(p=0.2234)$ descriptors. The perception of disability improved too, showing an amelioration trend in Health Assessment Questionnaire-HAQ $(p=0.4301)$ and Mouth Handicap in Systemic Sclerosis scale-MHISS$(\mathrm{p}=0.9775)$.

Conclusion: Our results confirm the potential efficacy of AFG to treat SSc skin complications and DUs. We reported an almost complete healing of DUs and a promising improvement of skin thickness and hardness both at hands and mouth, with good safety profiles. These clinical results were reflected by the subjective improvement of patients' wellbeing. Population enlargement and extended FU is ongoing to identify more responsive SSc subsets. Long term results with soft tissue biopsies will give us further data to finally evaluate the efficacy of this approach that aims to improve the care and quality of life of our SSc patients.

Disclosure of Interests: None declared

DOI: 10.1136/annrheumdis-2019-eular.5623

\section{THU0350 ANTI-INFLAMMATORY AND ANTI-FIBROTIC EFFECTS OF INTRAVENOUS ADIPOSE-DERIVED STEM CELL TRANSPLANTATION IN A MOUSE MODEL OF BLEOMYCIN-INDUCED SCLERODERMA}

Takayasu Suzuka ${ }^{1}$, Takuya Kotani ${ }^{1}$, Shogo Matsuda ${ }^{1}$, II Masaaki ${ }^{1}$ Tohru Takeuchi ${ }^{1}$, Shigeki Arawaka ${ }^{1}$, Koji Nagai ${ }^{1}$, Yumiko Wada' ${ }^{1}$ 'Osaka Medical College, Takatsuki Osaka, Japan

Background: Systemic sclerosis (SSc) is a systemic autoimmune disease characterized by microvascular damages and fibrosis. The main lesions of SSc are peripheral circulation insufficiency, sclerodermia, and interstitial pneumonia. Skin lesions are related to patient's ADL and QOL, however there is no effective treatment for normalizing the disease state. Adiposederived stem cells (AdSCs) have recently been considered a useful treatment tool for autoimmune disease because of their anti-inflammatory and immunosuppressive effects (ref).

Objectives: We investigated the therapeutic effect of intravenous mouse AdSCs (mAdSCs) transplantation in a SSc mouse model.

Methods: SSc was induced by bleomycin (BLM) in Balb/c mice, and the mice were assigned in the following groups: 1. Control (BLM-SSc), 2 mAdSCs $\left(1.0 \times 10^{4}\right.$ cells $), 3$. mAdSCs $\left(1.0 \times 10^{5}\right.$ cells $)$. After the administration of BLM, mAdSCs were injected via a tail vein on day 7. The mice were sacrificed at 14 days after mAdSCs injection, and the skins were harvested for histological analysis.

Results: In mAdSCs $\left(1.0 \times 10^{5}\right.$ cells) group, thickening of skin, hydroxyproline content, infiltration of inflammatory cells, gene expression of inflammatory cytokines, and fibrotic factors were significantly reduced compared with control group (Figure). But in mAdSCs $\left(1.0 \times 10^{4}\right.$ cells) group, there were no reduction of them. mAdSCs did not accumulate in skin. The levels of MMP-2, MMP-9, and COL1A1 mRNA expression at 21 days after BLM administration were significantly lower in mAdSCs $\left(1.0 \times 10^{5}\right.$ cells $)$ group compared with those in control group.

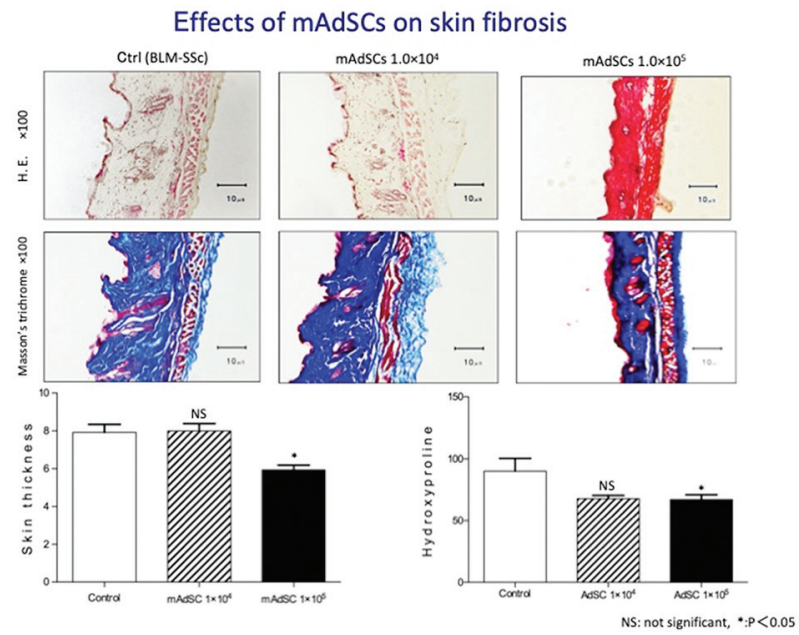

Abstract THU0350 - Figure 1

Conclusion: Intravenous mAdSCs inhibited both skin inflammation and fibrosis of BLM-SSc mice in a dose-dependent manner.

\section{REFERENCES :}

[1] Cell Transplant 2015.24: 2297-2305.

[2] Journal of Autoimmunity, 2016. 70: 31-39.

Acknowledgement: This study was supported by Grants-in-Aid for Scientific Research-KAKENHI- of Japan (Young Scientists B; 17K15761, 17K16216). 